\title{
Implementable Mechanisms for discrete utility functions, a solution using Tropical Geometry
}

\author{
Julián Enrique Chitiva Bocanegra *i†
}

August 9, 2019

\begin{abstract}
For mechanisms with two possible outcomes and a unique agent with two possible types, we state sufficient and necessary conditions over the type space that guarantees the existence of an incentive compatible mechanism. By using tropical geometry, we found that these conditions rely only on the relative valuations to be increasing over types. This result extends implementability for discrete utility functions.
\end{abstract}

JEL Classification: C62, C65, C79, D82, D86.

Keywords: Mechanism Design, Incentive compatible, Implementable mechanism, Tropical geometry.

*Advisor: Andrés Zambrano. An special acknowledgment to my parents for supporting me with my studies. In addition, I want to thank Andrés Zambrano, Paula Jaramillo, Çağatay Kayi and my friends for their comments and encouragement during this work.

${ }^{\dagger}$ Faculty of Economics, Universidad de los Andes, Bogotá D.C., Colombia. je.chitiva10@uniandes.edu.co

†Quantil, Bogotá D.C., Colombia. 


\section{Introduction}

Mechanism Design has been widely studied by economists. Using a pure algebraic or analytic approach has led to the solution of different imperfect information games and the demonstration of their properties. The important question involve the existence of mechanisms that are incentive compatible.

For a given type space, if the utility function defined in this space is continuous and differentiable of class $C^{2}$, Guesnerie and Laffont (1984) find that it is necessary that the single crossing condition holds to guarantee the existence of an allocation function that is incentive compatible. In addition, they show that if single crossing condition holds and the marginal rate of substitution is bounded, it is sufficient that the allocation function is monotone for the mechanism to be implementable. If an allocation function that is incentive compatible exists, the mechanism is implementable. In spite of the importance of this result, this only apply for a restricted class of type spaces because these conditions are difficult to obtain.

There have been two main attempts to extend the class of type spaces by relaxing the conditions proved by Guesnerie and Laffont (1984). First, Milgrom and Shannon (1994) show that the single crossing condition holds if and only if the utility function is supermodular and has increasing differences. This result extends the implementability of the allocation functions to utility functions that are not differentiable.

Second, Quah and Strulovici (2009) show that the single crossing condition defines an order over the space of utility functions. They also define the interval dominance order which is a weaker way to order utility functions and extend the class of type spaces for which there exists an implementable mechanism.

The contributions from Milgrom and Shannon (1994) and Quah and Strulovici (2009) are important because they show the existence of implementable mechanisms for a larger class of type spaces. Although the continuity of the utility functions defined 
by the type space remains necessary.

From a graph-theoretic approach, Rochet (1987) and Heydenreich et al. (2009) characterize incentive compatibility and revenue equivalence of mechanisms, respectively. First, Rochet (1987) studies a context of quasi-linear utility functions defined on a convex type space on $\mathbb{R}$ and differentiable valuations over the allocations. He shows that a mechanism is implementable if and only if for all cycles defined on the type space, the sum of differences of valuations when going through a cycle is non positive. Second, Heydenreich et al. (2009) define a relative valuation graph and proof that an allocation function satisfies revenue equivalence, hence implementability, if distances on this graph are antisymmetric.

The results from Rochet (1987) and Heydenreich et al. (2009) are important for the classical approach because they characterize implementability for any outcome space. However, their analysis relies on the differentiability of the valuations function.

In contrast, Crowell and Tran (2017) analyze the implementability of the mechanisms from the tropical spatial configuration of the agent's type. These authors provide the tools to verify if a mechanism is implementable or not. Nevertheless, they do not provide the necessary and sufficient conditions over these types to guarantee the existence of the mechanism. The machinery from tropical geometry used by them simplifies the analysis to depend only on the relative position of the agent's type in the tropical space, it also extends the analysis to discontinuous or discrete utility functions.

The purpose of this article is to use tropical geometry tools to find similar conditions to the ones given by Guesnerie and Laffont (1984), since this new framework simplifies the analysis. In this article, we focus our analysis in mechanism with one agent and two possible types. We show that it is necessary and sufficient that one of the types has a relative valuation for the second outcome greater than the other type. In spite of the importance of the revenue of a mechanism, we will not address mechanism efficiency problems in this article. 
This remainder of this article is organized as follows. In Section 2 we define the main framework and the problem that we analyze. We define the basic notions of tropical geometry to study mechanisms in Section 3. In Section 4 we provide a complete characterization of incentive compatible mechanisms and define the sufficient and necessary conditions over a type space of cardinality two to guarantee the existence of an incentive compatible mechanism. Finally, in Section 5 we conclude.

\section{Model}

The Tropical Real Space $(\mathbb{R} \cup \infty, \oplus, \odot)$ consider the real numbers with the infinity and defines two new operations, the tropical addition $(\oplus)$ which is the minimum in $\mathbb{R}, a \oplus b=\min \{a, b\}$, and the tropical multiplication $(\odot)$ which is the addition in $\mathbb{R}$, $a \odot b=a+b$. Here, $\mathbb{T P}^{1}$ is the natural compactification of the quotient space $\mathbb{R}^{2} / \mathbb{R} \odot \mathbf{1}$ called the tropical projective torus and is obtained from $\mathbb{R}^{2}$ by identifying vectors that differ from each other by tropical scalar multiplication. It is important to consider that we can identify $\mathbb{T P}^{1}$ with $\mathbb{R}$ via $\psi$

$$
\left(x_{1}, x_{2}\right) \in \mathbb{T P}^{1} \stackrel{\psi}{\mapsto}\left(x_{2}-x_{1}\right) \in \mathbb{R}
$$

Consider the following principal-agent model. A principal wants to design an Incentive Compatible (IC) mechanism with one agent with 2 known possible types. There are two possible outcomes or allocations. The truthful valuation $t^{*}$ is unknown by the principal. The agent type space is $T=\left\{t^{1}, t^{2}\right\}=\{(a, b),(c, d)\} \subset \mathbb{T P}^{1}$. Where the $i$-eth entry of $t^{j}$ represents type $j$ 's valuation for outcome $i$, see Table 1. We restrict our analysis for agents who cannot have an infinite valuation for an outcome, this restriction makes sense in the economic framework and it is not a strong restriction since valuations could be bounded independently of how big they are.

A mechanism is a pair $(g, p)$, consisting of an outcome function $g: T \rightarrow\{1,2\}$ and a payment function $p: T \rightarrow \mathbb{R}$. We will focus our analysis on private goods so the 


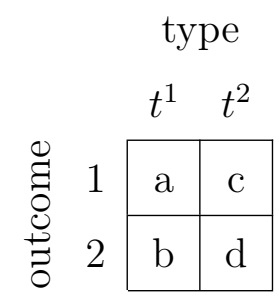

Table 1: Agent valuations.

outcome function is always assumed to be onto. Also, if the outcome function is not onto it would be a constant under this framework; therefore, trivially satisfies incentive compatibility. The agent's action is to declare to the mechanism a type $s \in T$, the game's outcome is $g(s)$, and the agent needs to pay $p(s)$. Remember that $s$ could be different from $t^{*}$. With this configuration, the agent's utility is

$$
u\left([g(s), p(s)], t^{*}\right)=t_{g(s)}^{*}-p(s) .
$$

Example 1. In the framework of the previous model, consider the following type space $T=\left\{t^{1}, t^{2}\right\}=\{(1,2),(0,3)\}$. Under this notation agent's valuations are

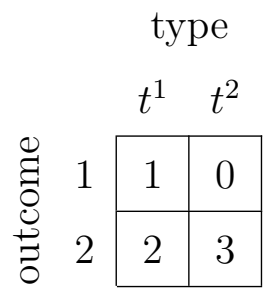

With this framework established, in the following sections, we will define the basic terminology needed for the analysis of mechanisms and complemented these notions with examples. Afterward, we determine the conditions over the type space that guarantee the existence of an implementable mechanism.

\section{Basic notions from tropical geometry}

With the model in mind, we are going to deconstruct it, to go to the basics of mechanism design from the tropical geometry perspective. First, we must understand what 
is an IC mechanism. And second, how can we obtain the set of IC outcome functions.

Definition 3.1 (Incentive compatible mechanism). A mechanism $(g, p)$ is said to be incentive compatible $(I C)$ if independently of $t^{*}$, the agent maximizes her utility by declaring her true type. This means that for a given $t^{*}, \forall s \in T$ the following inequality holds:

$$
\begin{aligned}
u\left(\left[g\left(t^{*}\right), p\left(t^{*}\right)\right], t^{*}\right) & \geq u\left([g(s), p(s)], t^{*}\right) \\
t_{g\left(t^{*}\right)}^{*}-p\left(t^{*}\right) & \geq t_{g(s)}^{*}-p(s)
\end{aligned}
$$

The outcome function $g$ is said to be incentive compatible or implementable if there exists a payment function $p$ such that $(g, p)$ is IC. We will use IC indistinctly from implementable, when referring to an outcome function.

Example 2. For the type space stated in Example 1, and because of the condition that the outcome functions must be onto, the only possible outcome functions are

$$
g^{1}=\left\{\begin{array}{ll}
1 & \text { if } t=t^{1} \\
2 & \text { if } t=t^{2}
\end{array} \quad g^{2}= \begin{cases}1 & \text { if } t=t^{2} \\
2 & \text { if } t=t^{1}\end{cases}\right.
$$

Note that this assertion is true for every problem of 2 types and 2 outcomes. So it is easy to generalize this result for every problem of two outcomes and two types.

Definition 3.2. Let $L^{g}$ to be the allocation matrix of a given outcome function $g$, this is an $m \times m$ matrix with $j k$-th entry

$$
L_{j k}^{g}=\inf _{t \in g^{-1}(j)}\left\{t_{j}-t_{k}\right\}
$$

It is important to note that we can represent an $n \times n$ matrix $A=\left(a_{i j}\right)$ by a weighted directed graph $G(A)$ with $n$ nodes labeled by $\{1,2, \ldots, n\}$. In $G(A)$ there is 
an edge from $i$ to $j$ if and only if $a_{i j}<\infty$ and we assign the length $a_{i j}$ to each such edge. It is important to consider the normalized length of a directed path from the node $i_{0}$ to the node $i_{k}$ defined as $\left(\sum_{j=1}^{k} a_{i_{j-1} i j}\right) / k$. A directed cycle is a directed path where $i_{0}=i_{k}$.

Example 3. For the outcome functions in the Example 2 the allocation matrices are

$$
\begin{aligned}
& L^{g^{1}}=\left(\begin{array}{cc}
0 & \inf _{t \in\left(g^{1}\right)^{-1}(1)}\left\{t_{1}^{1}-t_{2}^{1}\right\} \\
\inf _{t \in\left(g^{1}\right)^{-1}(2)}\left\{t_{2}^{2}-t_{1}^{2}\right\} & 0
\end{array}\right)=\left(\begin{array}{cc}
0 & -1 \\
3 & 0
\end{array}\right) \\
& L^{g^{1}}=\left(\begin{array}{cc}
0 & -1 \\
3 & 0
\end{array}\right) \rightarrow 0 \overbrace{-1} \\
& L^{g^{2}}=\left(\begin{array}{cc}
0 & \inf _{t \in\left(g^{2}\right)^{-1}(1)}\left\{t_{1}^{2}-t_{2}^{2}\right\} \\
\inf _{t \in\left(g^{2}\right)^{-1}(2)}\left\{t_{2}^{1}-t_{1}^{1}\right\} & 0
\end{array}\right)=\left(\begin{array}{cc}
0 & -3 \\
1 & 0
\end{array}\right) \\
& L^{g^{2}}=\left(\begin{array}{cc}
0 & -3 \\
1 & 0
\end{array}\right) \rightarrow 0 \overbrace{-3}
\end{aligned}
$$

Now, since agents cannot have infinite valuations, the directed graph associated to the allocations matrices is going to have a directed path between any two vertices and, therefore, be strongly connected.

Definition 3.3 (Tropical Eigenvalues and Eigenvectors). Let $A \in M_{n \times n}(\mathbb{R} \cup \infty, \oplus, \odot)$ an $n \times n-$ matrix with entries in the tropical real space. An eigenvalue of $A$ is $\lambda \in \mathbb{R}$ such that $A \odot x=\lambda \odot x$, for some $x \in \mathbb{R}^{n}$. Then $x$ is an eigenvector of the tropical matrix A.

Theorem 1. (Maclagan and Sturmfels, 2015) Let $A \in M_{n \times n}(\mathbb{R} \cup \infty, \oplus, \odot)$ whose graph $G(A)$ is strongly connected. Then $A$ has precisely one eigenvalue $\lambda(A)$. That eigenvalue equals the minimum normalized length of a directed cycle in $G(A)$ 
Theorem 2. (Crowell and Tran, 2017) An outcome function $g$ is IC if and only if the allocation matrix $L^{g}$ has tropical eigenvalue zero in the semiring $(\mathbb{R} \cup \infty, \oplus, \odot)$.

Proof. Although this Theorem is stated by Crowell and Tran, we offer an alternative proof. Fix $m \in \mathbb{N}, T \subset \mathbb{R}^{m}$ and suppose that $t^{*}$ is the true type of the agent. Let $g$ be an outcome function with allocation matrix $L^{g}$. Suppose that $j=g\left(t^{*}\right)$ and $l=g(s)$, where $s \in T$

( $\Longrightarrow$ ) Suppose that $g$ is IC. Define $x \in \mathbb{R}^{m}$ such that $x_{i}=p\left(g^{-1}(i)\right)$. Then

$$
\begin{aligned}
t_{g\left(t^{*}\right)}^{*}-p\left(t^{*}\right) & \geq t_{g(s)}^{*}-p(s) \\
t_{g\left(t^{*}\right)}^{*}-t_{g(s)}^{*}+p(s) & \geq p\left(t^{*}\right) \\
t_{j}^{*}-t_{l}^{*}+x_{l} & \geq x_{j} \\
\inf _{t \in g^{-1}(j)}\left\{t_{j}-t_{k}+x_{k}\right\} & \leq x_{j}
\end{aligned}
$$

Since this inequality holds $\forall k$ then $\inf _{t \in g^{-1}(i)}\left\{t_{i}-t_{k}+x_{k}\right\} \geq \min _{k}\left\{\inf _{t \in g^{-1}(i)}\left\{t_{i}-t_{k}\right\}+\right.$ $\left.x_{k}\right\} \geq x_{i}$, which must holds with strict equality. Then $L^{g}$ has min-plus tropical eigenvalue zero.

( $\Longleftarrow)$ Suppose that $L^{g}$ has min-plus tropical eigenvalue zero and min-plus tropical eigenvector $x$. Then $\forall i, \min _{k}\left\{\inf _{t \in g^{-1}(i)}\left\{t_{i}-t_{k}\right\}+x_{k}\right\}=x_{i}$ implies that $\forall i \inf _{t \in g^{-1}(i)}\left\{t_{i}-\right.$ $\left.\left.t_{k}\right\}+x_{k}\right\} \geq x_{i}$. In particular consider $i=j$, then $\left.\inf _{t \in g^{-1}(j)}\left\{t_{j}-t_{k}\right\}+x_{k}\right\} \geq x_{j}$ and therefore $t_{j}^{*}-t_{l}^{*}+x_{l} \geq x_{j}$. Now define $p: T \rightarrow \mathbb{R}$ by $p(t)=\pi_{g(t)} x$, where $\pi_{k}$ is the projection onto the $k$-th coordinate. Then

$$
\begin{aligned}
t_{j}^{*}-t_{l}^{*}+x_{l} & \geq x_{j} \\
t_{g\left(t^{*}\right)}^{*}-t_{g(s)}^{*}+p(s) & \geq p\left(t^{*}\right) \\
t_{g\left(t^{*}\right)}^{*}-p\left(t^{*}\right) & \geq t_{g(s)}^{*}-p(s)
\end{aligned}
$$

So $g$ is IC. 
By Theorem 2, the problem of determining the implementability of an outcome function is reduced to calculate the tropical eigenvalues of the allocation matrices. In Definition 3.3, we can see that the tropical eigenvalues of a matrix are simply the eigenvalues of the matrix but calculated using tropical operations. In addition, Theorem 1 states an easy way to calculate this tropical eigenvalue.

Example 4. From Example 3, consider the allocation matrix $L^{g^{1}}$ with their respective directed graph $G\left(L^{g^{1}}\right)$.

$$
L^{g^{1}}=\left(\begin{array}{cc}
0 & -1 \\
3 & 0
\end{array}\right) \rightarrow 0
$$

Now consider directed cycles on $G\left(L^{g^{1}}\right)$

\begin{tabular}{||c|c||c|c||}
\hline cycle & length & cycle & length \\
$\{1,1\}$ & 0 & $\{1,2,1\}$ & $\frac{-1+3}{2}=1$ \\
$\{2,2\}$ & 0 & $\{2,1,2\}$ & $\frac{3-1}{2}=1$ \\
\hline
\end{tabular}

Note that longer directed cycles are simply compositions of the shorter cycles in the table. Therefore, by Theorem 1, the tropical eigenvalue of $L^{g^{1}}$ is 0, and by Theorem 2 $g^{1}$ is implementable.

Example 5. From Example 3, consider the allocation matrix $L^{g^{2}}$ with their respective directed graph $G\left(L^{g^{2}}\right)$.

$$
L^{g^{2}}=\left(\begin{array}{cc}
0 & -3 \\
1 & 0
\end{array}\right) \rightarrow 0
$$

Now consider directed cycles on $G\left(L^{g^{2}}\right)$ 


\begin{tabular}{||c|c||c|c||}
\hline cycle & length & cycle & length \\
\hline$\{1,1\}$ & 0 & $\{1,2,1\}$ & $\frac{-3+1}{2}=-1$ \\
$\{2,2\}$ & 0 & $\{2,1,2\}$ & $\frac{1-3}{2}=-1$ \\
\hline
\end{tabular}

Note that longer directed cycles are simply compositions of the shorter cycles in the table. Therefore, since there is at least one cycle whose length is less than 0, by Theorem 1, the tropical eigenvalue of $L^{g^{2}}$ is less than 0, and by Theorem $2 g^{2}$ is not implementable.

\section{Conditions for implementability}

In Section 3 we established the tropical geometry basis to analyze the implementability and gave examples of IC and non-IC outcome functions. Specifically, from Examples 4 and 5, we can deduce a condition for an outcome function to be implementable, based on the associated allocation matrix.

First, note that in the graphs of the allocation matrix the two s $(i, i)$-cycles will always have a length of 0 since the allocation matrix has 0 on its diagonal. Then, by Theorem 1 in order to have a tropical eigenvalue of 0 , we only need that all remaining cycles have a strictly positive length. To fulfill the condition shown in Figure 1, we only need that the length $(a)$ of the path between nodes 1 and 2 is greater than the length $(b)$ of the path that returns from node 2 to node 1 , or vice versa, as we will prove in the Theorem 3 .

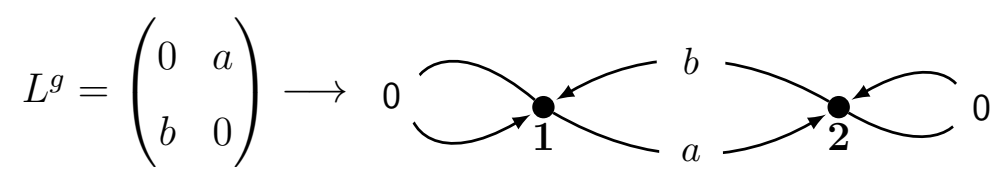

Figure 1: Condition over allocation matrix. 
Theorem 3. Consider the surjective outcome function $g: T \subseteq \mathbb{T} \mathbb{P}^{1} \rightarrow\{1,2\}$. Then $g$ is IC if, and only if, for the associated allocation matrix $L^{g}=\left(\begin{array}{ll}0 & a \\ b & 0\end{array}\right)$ it is true that $a+b \geq 0$

Proof. $\Longrightarrow$ Since $g$ is IC, by Theorem 2, $L^{g}$ has tropical eigenvalue 0 , therefore, by Theorem 1, the minimum normalized length of the directed cycles is 0 . Now, consider $L^{g}$ and $G\left(L^{g}\right)$

$$
L^{g}=\left(\begin{array}{ll}
0 & a \\
b & 0
\end{array}\right) \rightarrow 0
$$

Now consider directed cycles on $G\left(L^{g}\right)$

\begin{tabular}{||c|c||c|c||}
\hline cycle & length & cycle & length \\
\hline$\{1,1\}$ & 0 & $\{1,2,1\}$ & $\frac{a+b}{2} \geq 0$ \\
$\{2,2\}$ & 0 & $\{2,1,2\}$ & $\frac{b+a}{2} \geq 0$ \\
\hline
\end{tabular}

Since all the cycles that contains two or more nodes have normalized lengths greater or equal than $\frac{a+b}{2} \geq 0$, we conclude that $\frac{a+b}{2} \geq 0 \Longrightarrow a+b \geq 0$.

$\Longleftarrow$ Consider $L^{g}$ and $G\left(L^{g}\right)$

$$
L^{g}=\left(\begin{array}{ll}
0 & a \\
b & 0
\end{array}\right) \rightarrow 0 \overbrace{a}^{b}
$$

Now consider directed cycles on $G\left(L^{g}\right)$

\begin{tabular}{||c|c||c|c||}
\hline cycle & length & cycle & length \\
$\{1,1\}$ & 0 & $\{1,2,1\}$ & $\frac{a+b}{2}$ \\
$\{2,2\}$ & 0 & $\{2,1,2\}$ & $\frac{b+a}{2}$ \\
\hline
\end{tabular}

Since $a+b \geq 0 \Longrightarrow \frac{a+b}{2} \geq 0$ and longer cycles will have a greater or equal normalized length, since these are compositions of simpler cycles. We conclude 
that the minimum normalized length of a directed cycle is 0 . Therefore, by Theorem 2 and because $G\left(L^{g}\right)$ is strongly connected, $g$ is IC.

Note that, Theorem 3 states sufficient and necessary conditions over the allocation matrix, and more generally over $2 \times 2$ matrices with zero diagonal, which guarantees the implementability of the associated outcome function. Despite these conditions are similar to the ones obtained by Rochet (1987), his condition must be satisfied on all cycles, while this work inspects simple cycles (cycles with no subcycles). Second, we check that, by the construction of the allocation matrix, the characterization made by Heydenreich et al. (2009) is trivially satisfied.

The condition stated on Theorem 3 per se does not solve the problem of stating conditions over the type space. Observe that, to obtain these conditions, we must recover the type space from the allocation matrix.

Consider the diagram in Figure 2, First, note that the definition of $L^{g}$ gives us a mapping from the set of all possible type spaces of 2 -types, name $\mathcal{T}$, to the set of $2 \times 2$ matrices with zero diagonal, name $\mathcal{M}_{2 \times 2}$. Therefore, we need a mapping $\gamma$ from $\mathcal{M}_{2 \times 2}$ into $\mathcal{T}$, to state the desired conditions over the type space when restricting $\gamma$ to the set of matrices in $\mathcal{M}_{2 \times 2}$ which are associated to an implementable outcome function. Note that, $\mathcal{T} \subset \mathcal{P}\left(\mathbb{T P}^{1}\right)$ is the subset of the power set of $\mathbb{T P}^{1}$ whose elements has cardinality 2 .

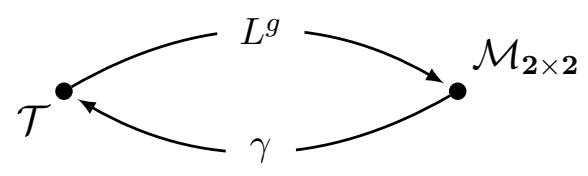

Figure 2: Mapping Diagram. 
There is an algorithmic way of constructing such $\gamma$. First, consider $L^{g}=\left(\begin{array}{ll}0 & a \\ b & 0\end{array}\right)$. Second, note that $L_{12}^{g}=a=\inf _{t \in g^{-1}(1)}\left\{t_{1}-t_{2}\right\} \Longrightarrow a=t_{1}-t_{2}, t \in g^{-1}(1)$ and $L_{21}^{g}=b=$ $\inf _{t \in g^{-1}(2)}\left\{t_{2}-t_{1}\right\} \Longrightarrow b=t_{2}-t_{1}, t \in g^{-1}(2)$. Third, since there is only two types, then $\left|g^{-1}(j)\right|=1$ and, without loss of generality, we can say that $a=t_{1}^{1}-t_{2}^{1}$ and $b=t_{2}^{2}-t_{1}^{2}$.

Fourth, recover the type space by defining $t^{1}=(a, 0)$ and $t^{2}=(0, b)$. Finally, since $t^{i} \in T \subset \mathbb{T P}^{1}$, the natural projection $\psi$ lies into $\mathbb{R}$ it is only important the relative valuations. So the types previously define are equivalent to $t^{1}=(0,-a)$ and $t^{2}=(0, b)$. So $\gamma: \mathcal{M}_{2 \times 2} \rightarrow \mathcal{T}$ is defined by $\left(\begin{array}{ll}0 & a \\ b & 0\end{array}\right) \stackrel{\gamma}{\mapsto} T=\left\{t^{1}=\left(\begin{array}{c}0 \\ -a\end{array}\right), t^{2}=\left(\begin{array}{l}0 \\ b\end{array}\right)\right\}$.

We can conclude that there exists an implementable outcome function $g$ if and only if $a+b \geq 0$, since this is a sufficient and necessary condition, over the allocation matrix, to determine whether the corresponding outcome function is implementable. Under the definition of the type space, the condition that $a+b \geq 0$ means that one of the types has a relative valuation for the second outcome, greater or equal than the other type, this result is consistent with the intuition from the economic theory.

\section{Conclusions}

The question now is, how this result is related to the previous results from the classical approach to implementable mechanisms? Since onto outcome functions trivially provides monotonicity, we were able to focus on which conditions over the type space were important. It is important to note that the condition that we found over the agent's valuation is similar to the increasing differences condition stated by Milgrom and Shannon (1994). Therefore for 2-types and 2-outcomes mechanisms, our result simplifies the analysis of implementability, reaching results similar to those obtained by the classical approach.

Finally, the potential uses of these results include the extension of the analysis to 
mechanisms that allocate public goods which consider non-onto outcome functions. Also, this result establishes the basis for the analysis of $n$-types and $m$-outcomes mechanisms.

\section{References}

Crowell, R. A. and Tran, N. M. (2017). Tropical geometry and mechanism design.

Guesnerie, R. and Laffont, J.-J. (1984). A complete solution to a class of principalagent problems with an application to the control of a self-managed firm. Journal of public Economics, 25(3):329-369.

Heydenreich, B., Müller, R., Uetz, M., and Vohra, R. V. (2009). Characterization of revenue equivalence. Econometrica, 77(1):307-316.

Maclagan, D. and Sturmfels, B. (2015). Introduction to Tropical Geometry, volume 161 of Graduate Studies in Mathematics. American Mathematical Society, Providence, RI.

Milgrom, P. and Shannon, C. (1994). Monotone comparative statics. Econometrica, $62(1): 157-180$.

Quah, J. K.-H. and Strulovici, B. (2009). Comparative statics, informativeness, and the interval dominance order. Econometrica, 77(6):1949-1992.

Rochet, J.-C. (1987). A necessary and sufficient condition for rationalizability in a quasi-linear context. Journal of mathematical Economics, 16(2):191-200. 